\title{
OPTIMIZATION OF THE URBAN LINE NETWORK USING A MATHEMATICAL PROGRAMMING APPROACH
}

\author{
L. JÁNOŠÍKOVÁ ${ }^{1}$, M. KOHÁNI ${ }^{1}$, M. BLATOŇ ${ }^{2} \&$ D. TEICHMANN ${ }^{2}$ \\ ${ }^{1}$ Department of Transportation Networks, University of Žilina, Slovak Republic. \\ ${ }^{2}$ Institute of Transport, VŠB-Technical University of Ostrava, Czech Republic.
}

\begin{abstract}
The paper deals with the line-planning problem related to urban public transport. Given the transportation network in a city, the origin-destination matrix of travel demands, and the fleet of available vehicles, the goal is to design the routes and frequencies of lines. The proposed solution method is a combination of the exact mathematical programming algorithm and a trip assignment procedure. The solution process consists of three stages: (i) initialization, (ii) designing the line network and setting the initial frequencies of selected lines, (iii) solution improvement. In the first stage, an initial set of feasible lines is proposed. In the second stage, an optimal subset of candidate lines is selected and initial frequencies of lines are computed by solving a mathematical programming model of the line-planning problem. The problem is formulated as a multiple criteria optimization problem, where the criteria reflect travelers' demand for a high quality service, operator's interest in an effective service, and the environmental impact of the vehicles. The solution of this problem specifies the number of vehicles of the given mode and type operating on the lines. The lines which are not assigned a vehicle will not operate. The assigned number of vehicles determines the frequency of a given line. At the same time, the solution specifies optimal passengers' routes in the line network. The third stage consists of an iterative process, which computes new line frequencies with regard to in-vehicle and waiting times, transfers, and passengers' behavior in a situation when they have multiple travel alternatives. The approach has been verified using real transportation data of a middle-sized city in the Slovak Republic. The paper presents the results of the case study.

Keywords: discrete choice model, line-planning problem, mathematical programming, multiple criteria optimization.
\end{abstract}

\section{INTRODUCTION}

The design of urban public transport lines should be addressed in the context of public transport planning process, which consists of five stages [1]:

1. designing the line routes,

2. setting the frequencies,

3. timetable development,

4. vehicle scheduling,

5. scheduling the drivers

The first two steps have a decisive influence on the quality of the whole transportation system. Line routes and frequencies determine how much the transportation demand is met because they have a direct impact on the spatial and time accessibility of the transportation service. The frequency of a line indicates the basic timetable period and controls the transportation capacity of the line. The route length determines the operating costs on the line, and together with the frequency it indicates the number of vehicles needed to operate on the line, which further results in investment costs. Thus line routes and frequencies are independent variables that influence two main objectives of the public transport design, namely the quality of the service as perceived by its users, and the operator's costs. 
In several previous approaches (e.g. [2]), the first two stages of the design (line routing and frequency setting) were solved simultaneously and were referred to as the line-planning problem (LPP). In our research we have followed this line of research considering the LPP as a multiple criteria optimization problem. We have proposed a new solution procedure that combines a mathematical programming approach with a trip assignment procedure. The solution process consists of three stages: (i) initialization, (ii) designing the line network and setting the initial frequencies of selected lines, (iii) solution improvement. In the first stage, an initial set of feasible lines is proposed. In the second stage, an optimal subset of candidate lines is selected and initial frequencies of lines are computed by solving a mathematical programming model of the line-planning problem. The problem is formulated as a multiple criteria optimization problem, where the criteria reflect travelers' demand for a high quality service, operator's interest in an effective service, and the environmental impact of the vehicles. The solution of this problem specifies the number of vehicles of the given mode and type operating on the lines. The lines that are not assigned a vehicle will not operate. The assigned number of vehicles determines the frequency of a given line. At the same time, the solution specifies optimal passenger routes in the line network. The third stage consists of an iterative process, which computes new line frequencies with regard to in-vehicle and waiting times, transfers, and passengers' behavior in a situation when they have multiple travel alternatives. This paper presents a mathematical programming formulation of the multi-criteria LPP and the solution procedure.

\section{PROBLEM STATEMENT}

The problem can be stated as follows: assume that the travel demand is given in the form of a so-called origin-destination (OD) matrix. The rows and columns of the matrix correspond to public transport stops. An element of the OD matrix determines the value of the transportation flow expressed in the number of travelers per time unit (e.g. an hour) who want to travel from the origin stop corresponding to the given row to the destination stop corresponding to the given column. Suppose that an initial set of all feasible lines has been pre-defined. The line network is not usually designed for an 'empty' city without any public transport, it is much more likely that we are supposed to improve an existing transportation system, and that is why the set of feasible lines can include current lines, modified current lines (e.g. lines avoiding overloaded roads), as well as new lines (e.g. corresponding to the shortest routes for the largest volumes of travelers). The task is to determine which lines from the candidate set will operate and to set their frequencies, so that the transportation service can be good and efficient.

The optimization criteria should be stated more precisely. First, let us look at the quality of transportation service from the passengers' point of view. Two main aspects of the transportation service perceived by its users are time and cost. The user's cost (fare) does not play a role in our line planning procedure because:

- Demand is treated as fixed and independent on the service quality offered between any origindestination pair. It means that a modal split is supposed to be done beforehand, and we deal only with those people who have decided to use public transport, so only that portion of the travel demand which is related to public transport inputs the problem.

- Costs of all routes are supposed to be the same.

So we can omit the fare and focus on time as the only passenger's criterion. If the travel demand refers to public transport stops, then travel time consists of three components: in-vehicle time, origin waiting time (the amount of time a passenger spends waiting for a bus at the origin stop), and 
transfer waiting time (the amount of time a passenger spends waiting during transfers). In-vehicle time depends proportionally on the line routes. Waiting times depend on line frequencies but this latter dependency is inverse proportional. In the mathematical model in-vehicle time is the only objective reflecting passengers' perception of quality. Waiting times are not modelled because then the model would be non-linear and difficult to solve [3]. Another reason why waiting times are not taken into account will be stated in the next section.

The second viewpoint regarding line planning is the efficiency of the provided service, more precisely operating and investment costs expended by the operator. In our model, investment costs do not perform an objective but are rather a constraint, which allows only the available vehicle fleet to be used for transportation service. As was explained in the introduction, operating costs depend directly on the route lengths and line frequencies, i.e. on the transportation output expressed in the number of kilometers ridden by all vehicles. Thus the transportation output will be the next objective in the mathematical model.

Another goal of the line design not mentioned so far is to minimize negative environmental impact of public transport, particularly electrical energy consumption, driving fuel consumption, and air pollution [4].

\section{MATHEMATICAL MODEL}

Input to the model includes a transportation network, travel demand, available vehicle fleet, and candidate lines.

A transportation network is modelled by a (di)graph $G=(N, A, t)$, where $N$ is a set of nodes (stops and road junctions) and $A$ is a set of feasible links, i.e. streets that candidate lines pass through. Every link $a \in A$ is associated with a driving time $t_{a}$. This time is in principle proportional to the length of the link but may also be affected by traffic volume in the link, and traffic organization, e.g. by traffic lights. We suppose that the driving time does not depend on the transportation mode neither on vehicle type. A vector of time distances is denoted by symbol $t$.

The travel demand is represented by an OD matrix $\boldsymbol{P}=\left\{p^{r s}\right\}$, where $p^{r s}$ indicates the number of travelers who want to travel in the considered time horizon from the origin stop $r$ to the destination stop $s$. The set of all OD pairs $(r, s)$ is denoted by symbol $Q$.

Vehicles of various modes and sizes can operate in the network. Let $I$ denote the set of available transportation modes (e.g. bus, trolley bus, tram). Vehicles of a given mode can be of different types, e.g. they can differ in the number of places, amount of engine emissions, energy consumption, etc. Let $J_{i}$ stand for the set of possible types for transportation mode $i \in I$. A vehicle of mode $i$ and type $j$ has $k_{i j}$ places and an operator can use at most $n_{i j}$ these vehicles. To make the model easy to understand and its explanation simpler, we reduce environmental impact to the major air pollutant, carbon monoxide (CO). Let $e_{i j l}$ be the amount of $\mathrm{CO}$ (given in grams) produced by one vehicle of mode $i$ and type $j$ per one $\mathrm{km}$ of line $l$.

Let $L$ stand for the set of all feasible lines. Line $l \in L$ is characterized by:

- its route with length $d_{l}$,

- transportation mode,

- upper frequency bound $f_{l}^{\text {max }}$, which depends on operational conditions, e.g. link or stop capacities for the given transportation mode,

- turnaround time $t_{l}^{\text {turn }}$ that defines how long it takes to drive line $l$ including stopping times at stops and layover time at the terminal point. The turnaround time is assumed to be independent on the vehicle type or loading. 
The set $L_{i} \subset L$ consists of the lines served by transportation mode $i$.

The goal is to decide which candidate lines from the set of feasible lines will operate, and to set their frequencies so that the quality of the service perceived by customers and economic efficiency is as good as possible and negative environmental impact of urban public transport could be minimal.

Both this decisions can be modelled by integer variables $x_{i j l}$, which determine how many vehicles of mode $i$ and type $j$ should be assigned to line $l$. Line $l$ that is not assigned a vehicle in the optimal solution (i.e. $x_{i j l}=0$ for all $j \in J_{i}$ ) will not operate. Using optimal values of variables $x_{i j l}$, frequency $f_{l}$ of line $l$ can be calculated as follows:

$$
f_{l}=\frac{1}{t_{l}^{\text {turn }}} \sum_{j \in J_{i}} x_{i j l}
$$

Most approaches reported in the literature (e.g. in [5-8]) use trip assignment as a pre-processing step. Assignment of vehicles to lines is calculated after the travel paths of the passengers have been fixed. Trip assignment is made using a shortest path method and results in the flow intensities on all links, which input the line-planning model as a parameter. Flow intensity $q_{a}$ on link $a \in A$ indicates how many passengers want to pass through link $a$ in the given time period. The model (used e.g. in [4]) contains a set of constraints assuring that the proposed lines will cover with a sufficient capacity all those links, which are used by at least one passenger (i.e. links with $q_{a}>0$ ).

A different approach can be found in [2]. Here optimal passenger routes are set in course of the solution process, it means that assignment of passenger flows to links is no more an input to the model but it becomes an output (a decision). This decision is represented by the variables $y_{a}^{r s} \in R_{0}^{+}$, which define how many passengers from $r$ to $s$ pass through link $a$. The total time that passengers traveling from $r$ to $s$ spend in a vehicle is expressed by the following term:

$$
\sum_{a \in A} t_{a} y_{a}^{r s}
$$

However, waiting times cannot be modelled because we do not know in advance the number of travelers who board or change lines at particular stops. For the same reason, even transfers of passengers are not modelled.

Using the above-mentioned variables, the mixed-integer programming model for the line-planning problem with multiple criteria can be formulated. In addition to the introduced notation, three other symbols occur in the model:

- $L_{a}-$ set of lines passing through link $a$;

- $A_{v}^{+}-$set of links outgoing node $v$;

- $A_{v}^{-}$- set of links ingoing node $v$.

Now the model can be written as:

$$
\begin{aligned}
\text { minimize } & \sum_{(r, s) \in Q} \sum_{a \in A} t_{a} y_{a}^{r s} \\
\text { minimize } & \sum_{i \in I} \sum_{j \in J_{i}} \sum_{l \in L_{i}} d_{l} x_{i j l}
\end{aligned}
$$




$$
\text { minimize } \sum_{i \in I} \sum_{j \in J_{i}} \sum_{l \in L_{i}} d_{l} e_{i j l} x_{i j l}
$$

subject to

$$
\begin{gathered}
\sum_{i \in I} \sum_{j \in J_{i}} \sum_{l \in L_{a}} \frac{1}{t_{l}^{\text {turn }}} x_{i j l} k_{i j} \geq \sum_{(r, s) \in Q} y_{a}^{r s} \text { for } a \in A \\
\sum_{a \in A_{r}^{+}} y_{a}^{r s}=p^{r s} \text { for }(r, s) \in Q \\
\sum_{a \in A_{s}^{-}} y_{a}^{r s}=p^{r s} \text { for }(r, s) \in Q \\
\sum_{a \in A_{v}^{-}} y_{a}^{r s}=\sum_{a \in A_{v}^{+}} y_{a}^{r s} \text { for }(r, s) \in Q, v \in N, v \neq r, v \neq s \\
\sum_{l \in L_{i}} x_{i j l} \leq n_{i j} \text { for } i \in I, j \in J_{i} \\
\sum_{j \in J_{i}} \frac{1}{t_{l}^{\text {turn }}} x_{i j l} \leq f_{l}^{\max } \text { for } i \in I, l \in L_{i} \\
y_{a}^{r s} \geq 0 \text { for }(r, s) \in Q, a \in A \\
x_{i j l} \in Z_{0}^{+} \text {for } i \in I, j \in J_{i}, l \in L_{i}
\end{gathered}
$$

The optimization criteria modelled by the objective functions (3)-(5) were described in Section 2. The objective function (3) stands for the total in-vehicle time of all travelers. The function (4) is the transportation output. The objective function (5) models the total amount of emissions produced by vehicles. The constraints (6) ensure that the total number of passengers traveling through link $a$ does not exceed the capacity of vehicles operating on that link. The constraints (7) and (8) respectively ensure that the travel demand will be satisfied. The constraints (9) are flow conservation equations in the nodes of the transportation network. The constraint set (10) limits the number of vehicles assigned to lines to the available amount. The constraints (11) bound the frequency of line $l$ to a maximum value given by operational conditions. The remaining obligatory constraints (12) and (13) specify the variable domains of the definition.

\section{SOLUTION METHODOLOGY}

The solution methodology proposed in this research was inspired by the approach published in [9] and detailed in [10]. The method consists of three stages. The first stage is an initialization, where a set of all feasible lines is designed. In the second stage, the multiple objective, mixed-integer programming problem (3)-(13) is solved by using a scalarization method. In the third stage, the solution of the problem (3)-(13) is improved. This stage consists of an iterative process, where new trip assignment and line frequencies are calculated in turn. The solution process is outlined in Fig. 1 and detailed in the following text. 
Scalarization is a traditional approach to solving multiple objective mathematical programming problems. The principle is that several objective functions are combined into a single objective function by means of a scalarizing function [11], and then a single objective problem with the same solution space is solved. The optimal solution of this problem is a compromise solution with regard to all objectives. We use Tchebycheff-norm scalarization that minimizes the weighed deviations of the particular objective functions from their ideal values [12].

The values of variables $x_{i j l}$ in the optimal solution of the problem (3)-(13) specify how many vehicles are assigned to the candidate lines. The lines that are not assigned a vehicle will not operate. The line frequencies are derived from the number of vehicles, using eqn (1).

The variables $y_{a}^{r s}$ specify passenger routes in the line network. Since the optimal solution of the problem (3)-(13) is a compromise with regard to all objectives, it is not optimal only from the

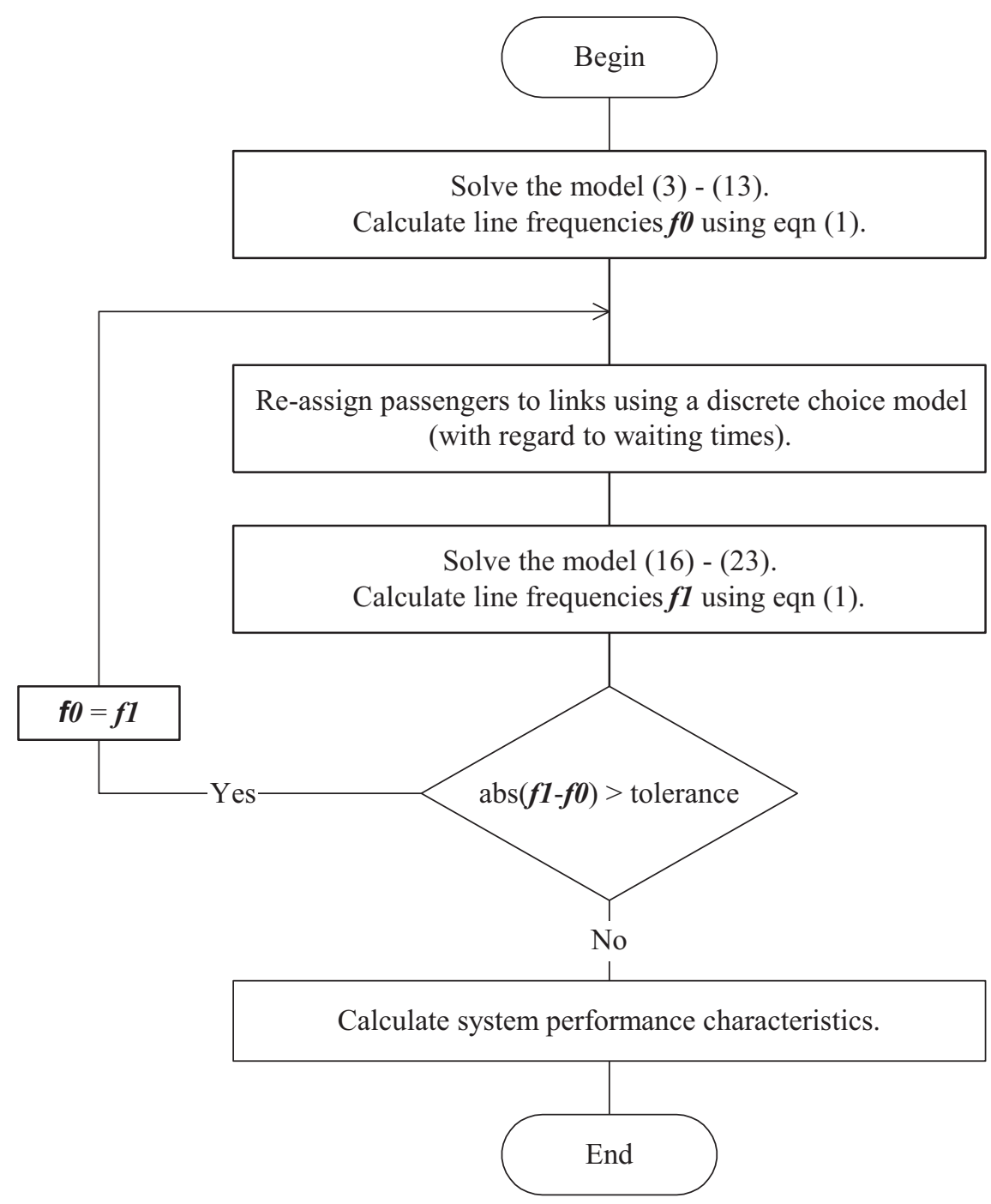

Figure 1: Flow chart of the solution process. 
travelers' point of view. Moreover, as it was mentioned above, neither waiting times nor transfers are modelled. Due to all these reasons, some travelers may be forced to take very long routes, possibly with a lot of transfers.

In the third stage of the solution process we try to improve the solution so that it can respect waiting times, transfers, and passengers' behavior in case there are multiple alternative paths between a given origin-destination pair. In this stage the line frequencies are adjusted repeatedly until the internal consistency of frequencies is achieved.

The stage involves an iterative process consisting of trip assignment and solution of a simplified mathematical programming model.

First a new assignment of travel demand to the proposed line network is computed, using a discrete choice model [13].

This trip assignment procedure should be described more precisely. Each origin-destination pair is considered separately. For a given $(r, s)$ couple, all feasible paths in the line network from stop $r$ to $s$ are found. The feasibility of a path is determined by two conditions: first, the path is a direct path or it contains at most a user-defined number of transfers; second, the travel time along this path does not exceed the minimum travel time by a specified threshold, say $30 \%$. The travel time consists of in-vehicle time, waiting time at the origin stop, and transfer waiting time. In case the line headway $\tau$ is less than 10 minutes, the average passenger waiting time at the origin stop can be estimated using the half headway model $[5,9,14]$. Otherwise the waiting time can be modelled by the term $2+0.3 * \tau$, where the time units are minutes [14]. The transfer waiting time is always $\tau / 2$ regardless of the headway. Moreover, a penalty for the transfer may be added to the travel time to model passengers' preference of paths without transfers.

If travelers from $r$ to $s$ can choose one of more feasible paths, a utility-based choice model is used to predict the number of passengers who will take a particular path. A traveler chooses a path with certain probability, which depends on the path utility. The utility has a deterministic and a random portion. The deterministic portion represents an observed measure of the path, while the random portion represents the randomness in the traveler's behavior, i.e. that component of the utility which is unknown to the analyst. In the context of urban public transport we can suppose that all of the feasible paths with the same origin and destination have the same cost. So it is only the travel time that represents the deterministic part of the path utility. From the family of utility-based choice models, the multinomial logit model can be used to choose a path. Let $K(r, s)$ denote the set of all feasible paths from $r$ to $s$ and $t_{i}^{\text {path }}$ stand for the travel time on path $i$. Then the number of passengers who will take the path $k$ on their trip from $r$ to $s$ is defined by eqn (14):

$$
p_{k}^{r s}=\frac{\exp \left(\mu t_{k}^{\text {path }}\right)}{\sum_{i \in K(r, s)} \exp \left(\mu t_{i}^{\text {path }}\right)} p^{r s}
$$

The coefficient $\mu$ in eqn. (14) is a parameter to be estimated. It has a negative sign which means that the path utility decreases with increasing travel time.

After the flows on all paths for all couples $(r, s)$ have been computed, the link flow $q_{a}$ on the link $a$ can be calculated as the sum of the flows on all paths that go through the link $a$.

The new link flows input a simplified mathematical programming model. The simplified model does not solve the passenger routes, it only computes the number of the vehicles to be assigned to the lines, i.e. line frequencies. It means that the model does not contain variables $\boldsymbol{y}$, objective (3), and constraints (7)-(9) and (12). As the link flows are known, another objective describing the quality of 
the transportation service perceived by passengers can be used instead of the traveling time. Such an objective is a comfort of a link. The comfort of a link is the ratio between supply and demand. Supply of a link is transportation capacity expressed by the number of places in vehicles that ride the given link per one time unit. Demand is the number of travelers of the given link per time unit, i.e. the link flow intensity. The goal is to maximize comfort of the worse link (link with the lowest ratio between supply and demand). For this reason we introduce a new variable $u$, which will denote the lowest comfort of a link of the transportation network. Then the constraints (6) will look as follows:

$$
\sum_{i \in I} \sum_{j \in J_{i}} \sum_{l \in L_{a}} \frac{1}{t_{l}^{t u r n}} x_{i j l} k_{i j} \geq q_{a} u \text { for } a \in A
$$

where $q_{a}$ is the total flow on link $a$.

The simplified model including the comfort element is again a multiple objective mixed-integer programming model:

$$
\begin{gathered}
\text { maximize } u \\
\text { minimize } \sum_{i \in I} \sum_{j \in J_{i}} \sum_{l \in L_{i}} d_{l} x_{i j l}
\end{gathered}
$$

subject to

$$
\text { minimize } \sum_{i \in I} \sum_{j \in J_{i}} \sum_{l \in L_{i}} d_{l} e_{i j l} x_{i j l}
$$

$$
\begin{gathered}
\sum_{i \in I} \sum_{j \in J_{i}} \sum_{l \in L_{a}} \frac{1}{t_{l}^{\text {turn }}} x_{i j l} k_{i j} \geq q_{a} u \text { for } a \in A \\
\sum_{l \in L_{i}} x_{i j l} \leq n_{i j} \text { for } i \in I, j \in J_{i} \\
\sum_{j \in J_{i}} \frac{1}{t_{l}^{\text {turn }}} x_{i j l} \leq f_{l}^{\max } \text { for } i \in I, l \in L_{i} \\
u \geq 0 \\
x_{i j l} \in Z_{0}^{+} \text {for } i \in I, j \in J_{i}, l \in L_{i}
\end{gathered}
$$

By solving the model (16)-(23), we get the new line frequencies. These new frequencies are compared with the previous ones, and if they differ by more than what a user-defined tolerance is, then a new trip assignment is performed and the whole process is repeated.

After the iterative process has finished, the system performance measures can be calculated. A decision maker is interested not only in particular criteria values but also in other characteristics, such as the average in-vehicle time (per a traveling person), the total and average waiting times, the total and average numbers of transfers, the minimum, maximum, and average line lengths expressed in the number of links and number of kilometers, and so on. 


\section{COMPUTATIONAL RESULTS}

The proposed model and solution methodology were verified by using the real data of public transport in the city of Žilina. Žilina is a middle-sized city situated in the northwestern part of the Slovak Republic. It has 85,302 inhabitants and covers the area of $80 \mathrm{~km}^{2}$.

The transportation service in the city is provided by the transportation operator Dopravný podnik mesta Žiliny (DPMŽ). During the day, the line network consists of 18 lines divided into 8 trolleybus lines and 10 bus lines. The line no. 99 is a special free bus line connecting the city center with the hypermarket Tesco. It operates from 9 a.m. to 10 p.m. At night, the city area is covered by 1 bus line. The scheme of the city line network is shown in Fig. 2.

DPMŽ has 89 vehicles at the disposal: 44 of the vehicles are Škoda-make trolleybuses produced in the years 1991-2001, and 45 of the vehicles are Karosa-make and Irisbus-make buses produced in the years 1990-2007. Tables 1 and 2 contain the basic summary of the vehicle fleet.

The travel demand (OD matrix) was derived from the statistical data provided by DPMŽ. The data are collected by the stamp machines, which are placed in vehicles and used to validate the passengers' travel tickets. The data have enabled us to find out how many travelers board a vehicle at each

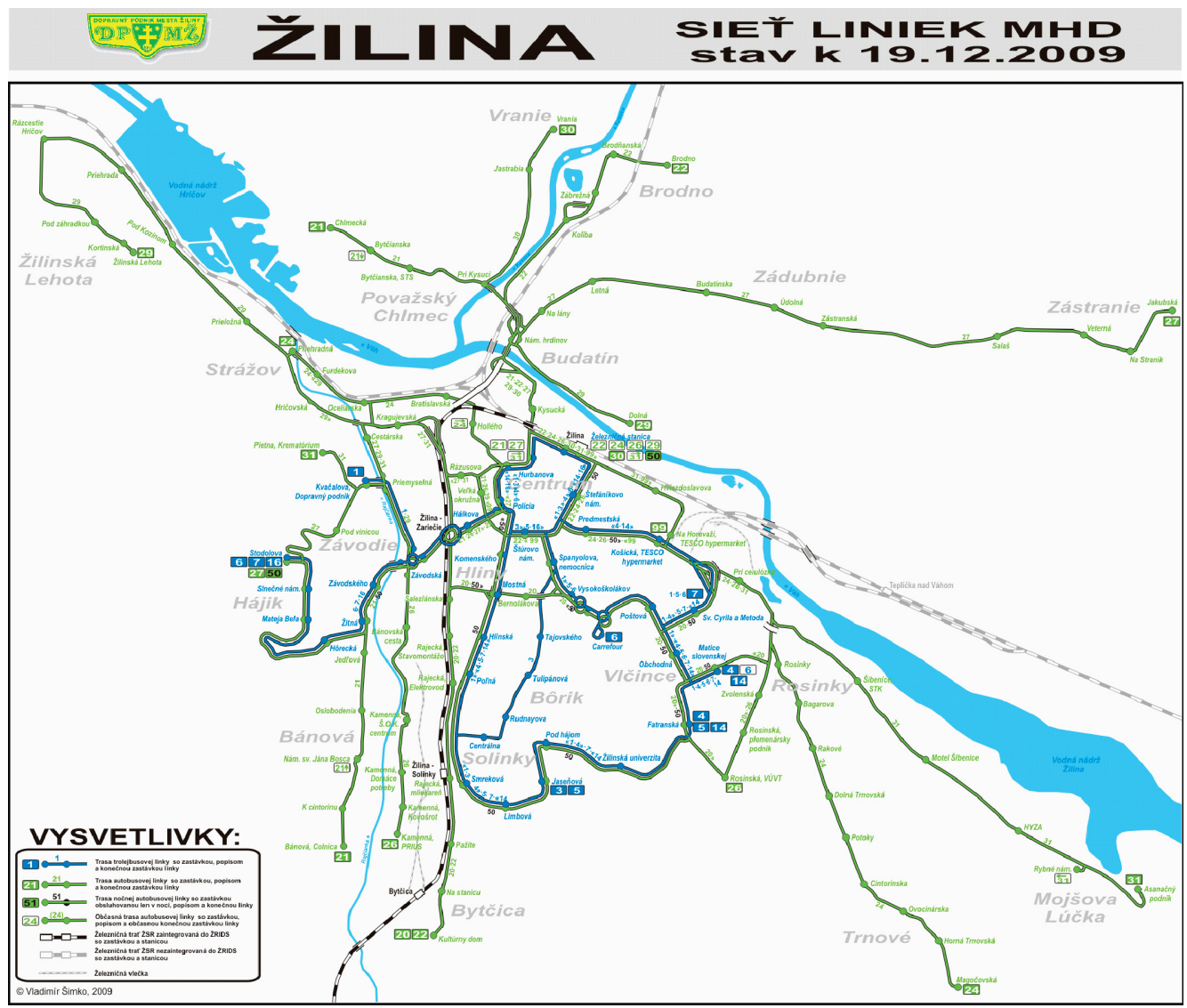

Figure 2: Line network in Žilina. 
stop during the investigated time horizon. It means that we know the number of trips generated in the origin stop $r$. Although we do not have the information on the destinations of the trips, they can be estimated from chip cards. When a passenger validates their electronic ticket at boarding, the stamp machine records the identification number of their chip card. We can assume that if the same card is used twice a day, and the interval between the first and the second validations is longer than an hour, then the origin stop of the second journey is the destination stop of the first journey. So we can estimate the direction distribution of the passengers boarding at stop $r$ (elements $p^{r s}$ of the OD matrix for the given origin $r$ and all destinations $s$ ).

Using the information from chip cards, the number of transfers between every origin stop and destination stop can be derived as well. This can help us calibrate the passengers' behavior model.

In the following paragraphs we present the experimental results for morning peak hours from 6:00 a.m. to 8:00 a.m. during a working day. For this time horizon, the OD matrix has 1,333 non-zero elements, which is approximately $21 \%$ of all of the elements (all possible couples of stops). The whole number of travelers in morning peak hours is 7,530. They perform 639 transfers. The number of transfers was used to estimate the parameters in the logit model: a path is regarded as feasible, if it has one transfer at most, the transfer penalty is 5 minutes, and $\mu=-1$.

All of the current lines were taken as feasible lines. Moreover, we have proposed a new trolleybus line connecting the housing estate Vlčince with the city center. This line corresponds to the shortest route for 3 out of 10 most intensive flows in morning peak hours. The network was pre-processed so that the memory and computational complexity of the model are reduced. Some rarely used stops between two neighboring stops were removed. Another condition necessary for the removal of a stop was that only one bus line would be stopping there, i.e. a transfer to another line was not possible there. The links conflicted with the removed stop were replaced by a single link connecting the neighboring stops. The line network with reduced stops and the new added line has 79 stops and 190 links. The other parameters of the model and solution method were set as follows:

Table 1: Characteristics of trolleybuses.

\begin{tabular}{lcc}
\hline Mark & Capacity (places) & Number of vehicles \\
\hline Škoda $14 \mathrm{Tr}$ & 83 & 13 \\
Škoda $15 \mathrm{Tr}$ & 150 & 17 \\
Škoda $15 \mathrm{TrM}$ & 150 & 14 \\
\hline
\end{tabular}

Table 2: Characteristics of buses.

\begin{tabular}{lccc}
\hline Mark & Capacity (places) & Emission level & Number of vehicles \\
\hline Karosa B 732 & 94 & EURO 1 & 9 \\
Karosa B 741 & 150 & EURO 2 & 1 \\
Karosa B 932 & 95 & EURO 2 & 6 \\
Karosa B 952 & 100 & EURO 4 & 17 \\
Karosa B 961 & 168 & EURO 4 & 3 \\
Renault City Bus PS09D1 & 100 & EURO 4 & 3 \\
Irisbus Citelis PS09D1 & 100 & EURO 4 & 3 \\
Irisbus Citelis PS09D2 & 96 & EURO 4 & 1 \\
Irisbus Citelis PU09D1 & 157 & EURO 4 & 2 \\
\hline
\end{tabular}


Upper frequency bound $f_{l}^{\text {max }}$ was set identically for all lines, namely eight vehicles per hour.

The tolerance in the stopping rule of the iterative process was set to zero as the experiments had proved that the process converged quite quickly, and after several steps the two successive frequency vectors were identical.

The mathematical model (3)-(13) with the described input parameters has 188,445 variables and 70,293 constraints. The model was implemented in the general optimization software Xpress-MP. The solution takes about 27 minutes on a personal computer equipped with the Intel Core 26700 processor with $2.66 \mathrm{GHz}$ and $3 \mathrm{~GB}$ of RAM.

The solution results are summarized in Table 3 . The performance characteristics for the current line network (in the first row denoted as March 2010) and several networks proposed by our mathematical programming approach are presented, namely the number of lines, number of vehicles needed, transportation output in vehicle-kilometers, overall quantities for all travelers (in-vehicle time, waiting time, number of transfers), the lowest comfort of passengers at a link, and the overall volume of emissions produced by all of the buses being used. The waiting time includes the time that passengers spend waiting at the origin and transfer stops. In-vehicle time, waiting time, and the number of transfers were computed using the presented passengers' behavior model.

The particular solutions presented in the second down to the fifth rows of the table are the following:

Opt. solution is the final result of the whole solution process described in Fig. 1.

Opt. solution (max. comfort) is the final solution optimized only in terms of passengers' comfort, i.e. it is the optimal solution of the problem: minimize (16) subject to (19)-(23) in the last iteration of the process described in Fig. 1.

Opt. solution (2nd stage) is the output of the second stage of the solution process, in which the initial line frequencies are computed, i.e. it is the optimal solution of the model (3)-(13).

Opt. solution (2nd stage, min. time) is the optimal solution of the second stage in terms of travel time, i.e. it is the optimal solution of the problem: minimize (3) subject to (6)-(13).

The optimal solution does not assign vehicles to the lines no. 1, 5, 6, and 7, which means that these lines are not supposed to operate in morning peak hours (see Table 4). The line no. 1 is a circular line interconnecting the housing estates Solinky, Vlčince and the city center. The line no. 5 is a similar line to approach Solinky and Vlčince from the city center and the other way around. In morning peak

Table 3: Results for morning peak hours 6:00-8:00 a.m.

\begin{tabular}{|c|c|c|c|c|c|c|c|c|}
\hline Network & Lines & Vehicles & $\begin{array}{l}\text { Output } \\
\text { [vehicle- } \\
\text { km] }\end{array}$ & $\begin{array}{c}\text { Total } \\
\text { in-vehicle } \\
\text { time }[\mathrm{h}]\end{array}$ & $\begin{array}{c}\text { Total } \\
\text { waiting } \\
\text { time }[\mathrm{h}]\end{array}$ & Transfers & $\begin{array}{c}\text { Comfort } \\
\text { [places per } \\
\text { passenger] }\end{array}$ & $\begin{array}{l}\text { Emissions } \\
\text { of } \mathrm{CO}[\mathrm{g}]\end{array}$ \\
\hline March 2010 & 17 & 37 & 648.94 & 1502 & 860 & 643 & NA & NA \\
\hline Opt. solution & 14 & 24 & 384.50 & 1491 & 1046 & 1077 & 1.83 & 1141.21 \\
\hline $\begin{array}{l}\text { Opt. solution } \\
\text { (max. } \\
\text { comfort) }\end{array}$ & 17 & 67 & 1250.10 & 1481 & 599 & 756 & 4.32 & 5752.99 \\
\hline $\begin{array}{l}\text { Opt. solution } \\
\text { (2nd stage) }\end{array}$ & 12 & 12 & 232.80 & 1531 & 2204 & 2053 & 0.28 & 872.67 \\
\hline $\begin{array}{l}\text { Opt. solution } \\
\text { (2nd stage, } \\
\text { min. time) }\end{array}$ & 15 & 58 & 1049.00 & 1503 & 757 & 1188 & 1.69 & 2626.54 \\
\hline
\end{tabular}


hours there is no interest in the transportation between the housing estates, and the other lines provide the transportation from the housing estates to the city center. There is a similar situation with the lines no. 6 (between the housing estates Hájik and Vlčince) and 7, connecting Hájik, Solinky, and Vlčince. The new added line (see the last row of Table 4) is intensively used: two employed vehicles provide almost four connections per hour.

Comparing the current line network to the optimal solution we can see that the efficiency criterion is more important in the mathematical model than in practice. As it was mentioned, the operator's costs are directly dependent on the transportation output, and the output depends on the number of vehicles used and on the line routes. The number of vehicles decreases in the optimal solution by $1 / 3$ (compared to the current state) and the output by $41 \%$. Despite these facts, the total in-vehicle time slightly decreases. On the other hand, lower number of vehicles assigned means lower line frequencies and then longer waiting time (by 22\%). Fewer vehicles and operating lines result into people having to change buses more frequently (the number of transfers increases by 67\%). Comfort and emissions cannot be evaluated for the present state because today vehicles are not fixed to lines but various types of vehicles can serve a line. This fact is expressed by the abbreviation NA (not applicable) in the corresponding cells of Table 3.

To set proper weight to the criteria, an expert to transportation planning needs to be involved in the planning process. In addition, another type of scalarization might be more suitable because the weights in Tchebycheff-norm scalarization are computed only from the coefficients in the objective function, and their role is to normalize particular criteria so that criteria values can be of the same order.

Table 4: Current and proposed frequencies for morning peak hours 6:00-8:00 a.m.

\begin{tabular}{lll}
\hline \multicolumn{3}{c}{$\begin{array}{c}\text { Current frequency } \\
\text { Line no. }\end{array}$} \\
\hline 1 & 1 & 0 \\
3 & 6 & 3.24 \\
4 & 5.5 & 4.86 \\
5 & 3 & 0 \\
6 & 3 & 0 \\
7 & 1.75 & 0 \\
14 & 5 & 4.74 \\
16 & 3.5 & 4.86 \\
20 & 1.75 & 1.25 \\
21 & 4 & 1.28 \\
22 & 3.5 & 2.18 \\
24 & 2.5 & 1 \\
26 & 2.25 & 1.03 \\
27 & 2 & 2 \\
29 & 0.5 & 1.05 \\
30 & 1 & 2.73 \\
31 & 0.75 & 1.03 \\
proposed & 0 & 3.87 \\
\hline
\end{tabular}


Comparing the final optimal solution (2nd row of Table 3) to the optimal solution of the complex model (3)-(13) in the fourth row proves the mentioned drawback of the model. The optimal solution of this model is a system optimum, and even more strongly than the final solution it accentuates the output at the expense of the quality of the service from the passengers' point of view. Therefore the third stage of the solution process is needed to get a solution that approaches demands of travelers more closely.

The other results of mathematical modeling are only informative, and they illustrate how the solution changes, if travelers' point of view is preferred.

\section{CONCLUSIONS}

The paper deals with the problem of planning the lines for urban public transport. The notion 'planning the lines' stands for the design of routes and frequencies of lines in correspondence with the terminology used in published literature. In our opinion, the contribution concerns two areas: modeling and methodology. We proposed two new multiple criteria mathematical programming models for the urban line network design. The exact algorithm solving the models is combined with a heuristic procedure with the aim to improve the quality of the design from the passengers' point of view.

The multiple criteria that have an impact on the final solution are: (i) the efficiency of transport, which is the primary requirement of an operator, (ii) the quality of the service perceived by passengers, which is quantified by in-vehicle time, waiting time, number of transfers, and travelling comfort, and (iii) the environmental impact expressed as the amount of emissions produced by buses. The criteria are in conflict because the operator's costs and emissions lead to minimizing the number of lines and vehicles, while travelers require as dense time and space schedules as possible.

The approach was verified using the case study of Žilina - a middle-sized city in the Slovak Republic. The computational results for morning peak hours are presented in the paper. The experiments show that a mathematical model can offer a high quality solution as the first proposal for an expert in transportation planning. The expert can further experiment with the solution and change the weights of the criteria so that a high-quality transportation service could be provided efficiently with a minimal negative environmental impact.

As far as we know, the environmental criterion has not been taken into account in previous studies related to line planning, though minimizing the environmental impact of the public transport is a necessary condition for sustainable mobility. Moreover we believe that a better public transport service will be more attractive for people and will cause a modal shift from cars to more ecological public transport.

\section{ACKNOWLEDGMENT}

This work has been supported by the Scientific Grant Agency of the Ministry of Education of the Slovak Republic and the Academy of Sciences under project VEGA 1/0361/10 Optimal designing of public service systems under uncertainty.

\section{REFERENCES}

[1] Ceder, A. \& Wilson, N.H., Bus network design. Transportation Research Part B, 20(4), pp. 331-344, 1986. doi: http://dx.doi.org/10.1016/0191-2615(86)90047-0

[2] Borndörfer, R., Grötschel, M. \& Pfetsch, M.E., A column-generation approach to line planning in public transport. Transportation Science, 41(1), pp. 123-132, 2007. doi: http://dx.doi. org/10.1287/trsc. 1060.0161

[3] Erlander, S. \& Schéele, S., A mathematical programming model for bus traffic in a network. Proc. of the 6th Int. Symposium On Transportation and Traffic Theory, ed. D.J. Buckley, Elsevier: New York, London, Amsterdam, pp. 581-605, 1974. 
[4] Blatoň, M., Vícekriteriální optimalizace linek MHD. Proc. of the Seminar Discrete Optimisation Problems in Transportation. University of Pardubice: Pardubice, Czech, 2009.

[5] Černý, J. \& Kluvánek P., Základy matematickej teórie dopravy, VEDA: Bratislava, Slovak, 1991.

[6] Peško, Š., Podpora metód operačného výskumu pri navrhovaní systému liniek. Proc. of the 6th Int. Conf. On Urban Transportation Infrastructure. University of Žilina: Žilina, Slovak, 2008.

[7] Surovec, P., Provoz a ekonomika silniční dopravy I. Vysoká škola báňská: Ostrava, Czech, 2000.

[8] Teichmann, D., O několika modifikacích matematického modelu přidělování vozidel linkám v městské hromadné dopravě. New Railway Technique, 17(1), pp. 20-23, 2009.

[9] Fan, W. \& Machemehl, R.B., Optimal Transit Route Network Design Problem: Algorithms, Implementations, and Numerical Results. Report No. SWUTC/04/167244-1. Center for Transportation Research: University of Texas at Austin, 2004.

[10] Jánošíková, L., Blatoň, M. \& Teichmann, D., Design of urban public transport lines as a multiple criteria optimisation problem. Proc. of the 16th International Conference on Urban Transport and the Environment - Urban Transport XVI, eds A. Pratelli \& C.A. Brebbia, WIT Press: Southampton, pp. 137-146, 2010.

[11] Ehrgott, M. \& Wiecek, M.M., Multiobjective programming (Chapter 17). Multiple Criteria Decision Analysis: State of the Art Surveys, eds J. Figueira, S. Greco \& M. Ehrgott, Springer: New York, 2005.

[12] Fiala, P., Modely a metody rozhodování. Oeconomica: Praha, Czech, 2006.

[13] Koppelman, F.S. \& Bhat, C., A Self Instructing Course in Mode Choice Modeling: Multinomial and Nested Logit Models, 2006, available at http://www.civil.northwestern.edu/people/koppelman/PDFs/LM_Draft_060131Final-060630.pdf

[14] Fan, W. \& Machemehl, R.B., Characterizing bus transit passenger waiting times. 2nd Material Specialty Conference of the Canadian Society for Civil Engineering. Canadian Society for Civil Engineering: Montreal, 2002. 